\section{A Taxonomic Research Update of Cultivated Liriopogons}

\author{
Paul R. Fantz
}

Additional index words. nomenclature, Liriope, Ophiopogon, Convallariaceae, Liliaceae, ornamental, ground covers

Summary. A taxonomic revision of liriopogons (Liriope Lour., Ophiopogon Ker-Gawl) cultivated in the United States is in progress at North Carolina State Univ. Germplasm was obtained from nurseries, botanical gardens/arboreta, and private collectors. Nearly $17 \%$ of the germplasm was misidentified to genus; nearly $36 \%$ misidentified to species; and nearly $14 \%$ received under one name from one source contained mixed germplasm. Preliminary analysis of data indicate a minimum of five species of Liriope and eight species of Ophiopogon are in cultivation. Six additional taxa have not flowered. Polygonal graph analysis was used to visualize biometrical data and observe relationships among taxa. Additional taxonomic publications for segregation of genera and species of liriopogons, including an inventory of taxa, quantitative descriptions, illustrations, and keys, are in progress.

Department of Horticultural Science, North Carolina State University Box 7609, Raleigh, NC 27695-7609.

\section{${ }^{\prime}$ Professor.}

Research Project NCO 6104 funded in part by the North Carolina Agricultural Research Service, Raleigh, NC 27695-7643. Thanks and appreciation to those who contributed to the germplants collection of liriopogons: especially J. C. Raulston, Director, North Carolina State Univ. Arboretum, Raleigh; Gary Adams, Evergreen Nursery, Atlanta, Ga.; Ken and Belle Durio, Louisiana Nursery, Oelousas, La.; Mike Sinnott, Assistant Curator and J.L.S. Keesing, Scientific Liason, Living Collections, Royal Botanic Gardens, Kew, U. K.; and Clarence Landis, Spanish Fort, Ala. Appreciation is extended to Paul Lindberger, Superintendent, Horticultural Field Laboratory, Raleigh, forpreparation of the research beds, shade stricture, and weed maintenance. Thanks to Frank Blazich for his critical review of the manuscript. Use of nursery and trade names in this publication does not imply endorsement by the NCARS of the nurseries or the products mentioned nor criticism of similar ones mentioned. The cost of publishing this paper was defrayed in pan by the payment ofpage charges. Under postal regu lations, tbis paper therefore must be hereby marked advertisement solely to indicate this fact.
$\mathrm{L}$ iriopogons (Liriope Lour., Ophiopogon Ker-Gawl) cultivated in the United States have complex taxonomic problems that need to be resolved (Fantz, 1993). These include: 1 ) an inadequate inventory of cultivated liriopogons, 2) a lack of quantitative descriptions of taxa, 3) the absence of keys to aid identification, 4) a lack of vouchers for morphological comparison, 5) the inaccessibility and scarcity of Asian floras and lack of liriopogon revisions in the literature, and 6) nomenclatural questions raised from the classification level of family to cultivar. Nursery practices of sexual propagation of cultivars, plant substitution, mislabeling of cultivars, and seedling invasion into cultivar stock lead to cultivar degradation that makes cultivar differentiation difficult.

Research was initiated at North Carolina State Univ. to address taxonomic problems associated with cultivated liriopogons (Fantz, 1993). The objective was to accumulate germplasm representative of all taxa of liriopogons cultivated in the United States, grow them together for morphological comparison, and prepare a taxonomic revision of liriopogons cultivated in the United States.

\section{Materials and methods}

Germplasm of liriopogons for this taxonomic research was obtained by purchased acquisition from nurseries, donations by nursery operators, liriopogon collectors and botanic gardens / arboreta, and through the plant exchange program of the North Carolina State Univ. Arboretum (Table 1). Germplasm of liriopogon is sold commonly in the nursery-landscape trade by "bibs," a term lacking in modern horticultural references (e.g., Bagust, 1992; Huxley, 1992). A bib is "a shoot or plantlet consisting of one crown with leaves and attached roots and/or stolons" according to a compilation of definitions from nursery operators.

Each taxa received was potted using a medium consisting of 2 potting soil :1 sand :1 peatmoss :1 bark humus (by volume) and placed in a greenhouse. One bib was maintained in the greenhouse as a stock plant. The other bibs were transplanted in the spring in beds at the Horticultural Field Laboratory, Raleigh. Beds were prepared by tilling the soil (Piedmont clay), fumigating, and adding $5 \mathrm{~cm}$ of ground pine bark mulch. The beds were covered with a $55 \%$ shadecloth. Individual plants were tracked by a coding system.

A data sheet was prepared that included 78 morphological characters, 36 vegetative characters, and 42 reproductive characters. Herbarium vouchers were made of each collection at different stages of growth. Data were recorded from 947 herbarium vouchers of liriopogons and from observations and measurements obtained from live plants. Parallel quantitative descriptions of each taxa were prepared from these morphological data.

Polygonal graph analyses were made of similar taxa. Polygonal graphs are used to compare graphically the variation patterns within a taxa (Radford et al., 1974). This taxonomic tool permits visualization of biometric measurements and observation of differences or similarities. Both qualitative (e.g., flower color) and quantitative (e.g., leaf width) characters can be used. A series of axes are drawn equidistant through a central point. A minimum of reliable, segregating, morphological characters are chosen and plotted on the axes. Characters can be plotted by points on each axis, representing measurements, or by coded values whose distances represent a qualitative character (e.g., clumping, stoloniferous) or a range of measurements (e.g., narrow leaves, 1 to $5 \mathrm{~mm}$; medium leaves, 5 to $7 \mathrm{~mm}$; broad leaves, 7 to $11 \mathrm{~mm})$. Each plotted

Table 1. An inventory of names of cultivated liriopogon species in research study.

\begin{tabular}{ll}
\hline Liriope sp. & \multicolumn{1}{c}{ Ophiopogon sp. } \\
\hline L. exiliflora & O. arabicus \\
L. gigantea & O. bockianus \\
L.graminifolia & O. bodineri ${ }^{\mathrm{z}}$ \\
L.grandiflora & O. bodinieti ${ }^{\mathrm{z}}$ \\
L. kansuensis ${ }^{2}$ & O. chekangensis \\
L. minor & O. clarkei \\
L. muscari & O.graminifolia \\
L. platyphylla & O. intermedius \\
L. spicata & O. jaburan \\
& O. japonicus \\
& O. marmoratus ${ }^{\mathrm{z}}$ \\
& O. nana \\
& O. nigra \\
& O. nigrescens \\
& O. ohwii \\
& O. parpiflorus ${ }^{2}$ \\
& O. planiscapus \\
& O. spicatus \\
& O. wallichianus \\
& \\
&
\end{tabular}

${ }^{2}$ Recent accessions. 
point on art axis is connected by straight lines, resulting in a polygonal image of the taxa. These polygons can be compared with polygons of other taxa for similarities and differences.

\section{Results and discussion}

Germplasm accumulated for this project currently includes 22 named species (Table 1), eight labeled as Liriope, and 13 labeled as Ophiopogon. There are 88 labeled cultivars (Table 2). Four bibs were obtained for most taxa. Only one or two bibs of lesscommon clones and species could be obtained from sources because of the scarcity of the taxa and because of the higher cost ( $\$ 15$ to $\$ 26$ per bib). Bibs received for this study from nursery operators varied in size from one plantlet with juvenile leaves 2 to $5 \mathrm{~cm}$ long with sparse roots, to several plantlets with mature leaves and well-developed root systems.

Morphology. Some accessions grew slowly, taking up to 4 years to bloom. Not all collections have flowered: some flowered. but did not set fruit. Reproductive material is required for accurate identification of taxa. Therefore, current descriptions for some accessions of liriopogons are incomplete. These accessions must be grown for one or more additional years in order for reproduction to occur and provide the necessary morphological structures needed for data accumulation.

Comparison and analysis of avail- able morphological data indicated a minimum of 13 species in the trade, five species of Liriope, and eight species of Ophiopogon. Two distinguishable species of Ophiopogon currently lack an identification. Recent acquisitions from arboreta could add possibly six more different species (Table 1, recent accessions), or help in the identification of the two unknowns.

Misidentifications. Many liriopogons look similar vegetatively, and therefore are difficult to distinguish. Many nursery operators commonly define Liriope as growing taller with broader leaves and Ophiopogon as smaller in stature with narrower leaves. This is not accurate; therefore, errors in identification are expected. To date, $17 \%$ of the germplasm studied was misidentified to genus. Most of these misidentifications were species of Ophiopogon labeled as a Liriope. Commonly confused were Liriope spicata Lour. with Ophiopogon japonicus (Thunb.) Ker-Gawl., and Liriope muscari (Decne.) L.H. Bailey with "Aztec grass," an Ophiopogon as first reported by Adams (1989). Plants obtained for this study sold as $L$. kansuensis C.H. Wright and L. graminifolia Hort. were Ophiopogon. Ophiopogon planiscapus Nak. occasionally was misidentified as L. spicata.

Nearly $36 \%$ of the germplasm was labeled with the wrong specific epithet. Four names are commonly used in the trade: L. muscari, L. spicata, O.

Table 2. An inventory of names of cultivated liriopogon cultivars in the research study.

\begin{tabular}{llll}
\hline Alba & Green Midget & Monroe & Silvery Dragon \\
Arabicus & Gyoku-Ryu & Monroe White & Silvery Sunproof \\
Aritaki & Hakuryu & Moores White & Sunproof \\
Aztec & Hakuryu Dwarf & Nana & Superba \\
Big Blue & Hakuryu Ko & New Orleans & Tamaryu Nishiki \\
Big Blue Regal & Hawks Feather & New Wonder & Tidwells Big Blue \\
Blue Cushion & Improved Muscari & Nigrescens & Torafu \\
Blue Spire & Ingwersen & Original Big Blue & Traebert White \\
Border Gem & Intermedia & Peedee Ingot & Variegata \\
C.T. Tanner & Jeanerette & Platypus & Variegata Alba \\
Christmas Tree & John Burch & Purple Bouquet & Variegata White \\
Crows White & Kigimafukiduma & Regal & Variegated \\
Crested White & Korean Variegated & Rocket & Variegated Ever- \\
Curly Twist & Kioto & Royal Purple & green Giant \\
Densiflora & Korean Variegated & Samantha & Variegated White \\
Ebony & Kyoto Dwarf & Sheffield & Flowered \\
Ebony Knight & Lilac Beauty & Shiro Tama Hime & Variegatus \\
Evergreen Giant & Magestic & Shiroshima Ryu & Webber \\
Franklin Mint & Majectic & Silver Banded & Webster Wideleaf \\
Gilner White & Majestic & Silver Dragon & White on White \\
Gold Banded & Majestic Variegated & Silver Mist & Wonder Evergreen \\
Grandiflora & Miniature & Silver Showers & Xmas \\
Grandiflora White & Minor & Silvery Midget & \\
\hline & & &
\end{tabular}

planiscapus, and O. japonicus. Plants acquired as L. muscari in this study were a mixture of three species. Plants acquired as $O$. japonicus included four distinct species. In addition, L. muscari was misidentified as L. spicata. Ophiopogon planiscapus is distributed under the names $O$. arabicus Hort., O. nigra Hort. and $O$. nigrescens Hort. The latter three names are illegitimate.

Nearly $14 \%$ of the germplasm from one source under one name was improperly identified. For example, one accession (87-022 ), labeled as 'Lilac Beauty', contained four bibs. One bib had purple flowers with the erect flowering stalked unbranched (= 'Lilac Beauty'), whereas two bibs had pinkish flowers and a branched axis in the flowering stalk (= 'Christmas Tree'). The fourth bib died before flowering.

These errors of selling mixed germplasm under one name may result from misidentification of stock material, or from nursery cultural practices (sexual reproduction of clones, plant substitution, seedling invasion, labeling practices), as defined by Fantz (1993). It is obvious from material received for this study that accurate germplasm identification of liriopogons is a major problem in the nurserylandscape industries.

Polygonal graphs. Morphological data indicated that four species are being sold under the name $O$. japonicus. Polygonal graphs were prepared using vegetative and flowering characters to visualize data for these species (Fig. 1). Polygonal graphs $1 \mathrm{~A}$ to $1 \mathrm{C}$ are impostors, quite dissimilar polygons to 1D, which represents the true O. japonicus.

Polygonal graphs (Fig. 2) were prepared using foliar and reproductive characters for four variegated cultivars reported as L. muscari. Adams ( 1989) reported much confusion over 'Silvery Sunproof', which is regarded as distinct from 'Variegata', but often has been confused with it. The polygonal graphs 2A ('Silvery Sunproof) and 2B ('Vanegata') substantiate the close relationships of these two clones, as the polygons are very similar in shape.

'Sunproof is regarded as an improved selection derived from 'Silvery Sunproof. The polygonal graphs 2A and 2C (Fig. 2) indicate a remarkable difference in nearly all characters plotted raising skepticism that 'Sunproof (2C) is derived from 'Silvery Sunproof' (2A). The main similarity appears to be only in name and the variegated 

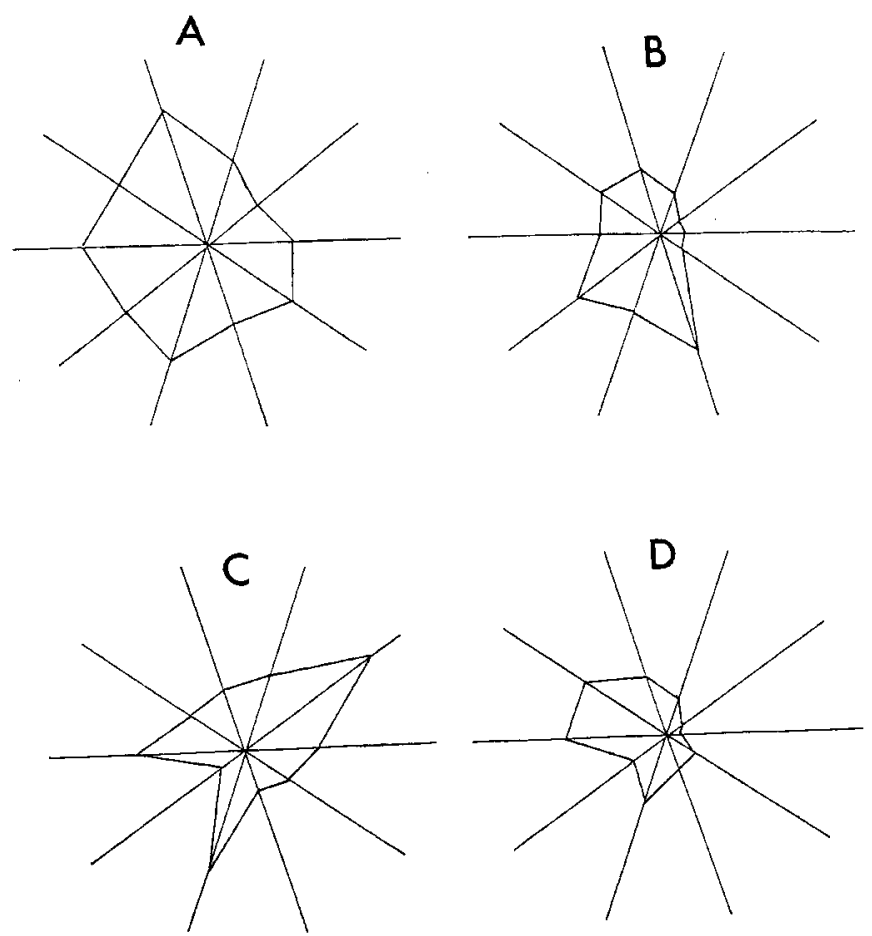

Fig. 1. Polygonal graphs of four species sold under the name of Ophiopogon japonicus. Ten axes counterclockwise from Lefi horizontal represent style length, perianth lobe length, leaf length, leaf width, average scape length, average rachis length, pedicel length, number of fascicles, foliar bract length, and pevianth tribe length, respectively. (A) Imposter \#1; (B) Imposter \#2; (C) Importer \#3; (D) Ophiopogon japonicus.
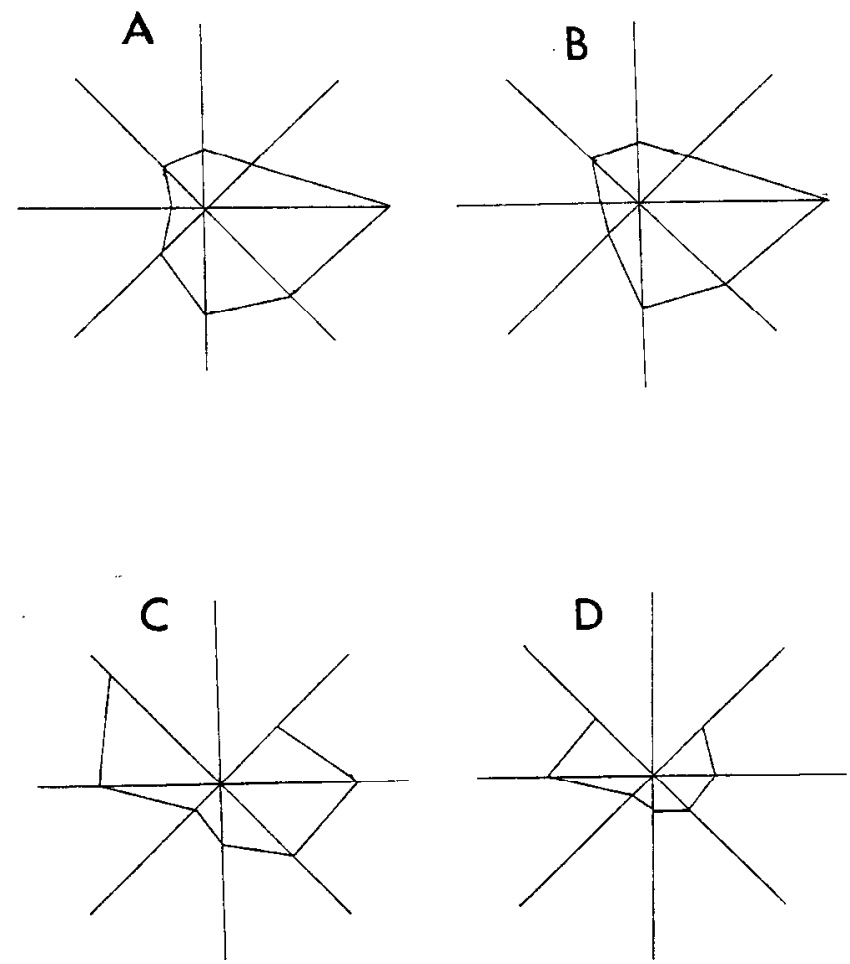

Fig. 2. Polygonal graphs of four variegated cultivars sold under the name of Liriope muscari. Eight axes counterclockwise from left horizontal represent peduncle rachis difference, bud persistence, fruit diameter,.growth habit, average scape length, avevage peduncle length, average vachis length, and flower duration, respectively. (A) 'Silvery Sunproof; (B) 'Variegata'; (C) 'Sunproof; (D) 'Silver Midget'.

foliage. An unexpected relationship was discovered when a polygonal graph was prepared for 'Silvery Midget' (2D) and compared to these other varie- gated clones. The polygon suggests that 'Silvery Midget' may be a dwarf selection of 'Sunproof'.

Polygonal graphs are being re- vised using different morphological characters, as the database expands to obtain additional relationships. Characters used and the number of axes required also will change depending on the similarity of taxa and the classification level being compared.

Taxonomy. Cultivated liriopogons present a complex taxonomic problem. The magnitude of the problem has been a major obstacle since initiation of this research, particularly with new taxa entering the trade each year. Another major obstacle has been accurate species determination. Liropogons are native to Asia, where floras are unavailable, hard to access when available, and sometimes difficult to translate when obtained. The number of impostors within species was a surprise. Morphologically, they can be segregated, but not all have been identified accurately with a name.

It is difficult to provide an inventory of cultivars when the problem with species segregation and identification is not completed. Originally, 25 to 30 cultivars were expected, but 89 named cultivars have been collected.

\section{Conclusion}

A taxonomic revision of liriopogons cultivated in the United States is currently in progress. Almost all cultivars in the study can be assigned to one of 13 morphologically defined species. Potentially, six additional species, not yet in the trade but available in aboreta collections, need further study. Further macromorphological research will be continued on cultivars and newer taxa to provide an inventory of cultivated liriopogons, quantitative descritions, and keys to identification of taxa.

\section{Literature Cited}

Adams, G. 1989. Great ground covers. Amer. Nurseryman 170(8):83-91.

Bagust, H. 1992. The gardener's dictionary of horticultural terms. Cassell, London.

Fantz, P.R. 1993. Taxonomic problems in cultivated liriopogons. HortTechnology 3(2):146-150.

Huxley, A. (ed.). 1992. The new Royal Horticultural Society dictionary of gardening. vol. 3. Morton Word Processing, Scarborough, U.K.

Radford, A. E., W. C. Dickison, J.R. Massey, and C.R. Bell. 1974. Vascular plant systematics. Harper \& Row, New York. 